\title{
Novel Histological Evidence of Collagen and Elastin Regeneration in Fractional RF-Treated Acne Scars
}

\author{
Judith Hellman ${ }^{*}$, Jonathan Yao² \\ ${ }^{1}$ Mt. Sinai Hospital, New York, USA \\ ${ }^{2}$ Schweiger Dermatology Group, New York, USA \\ Email: `jhderm@gmail.com, jonathan.YaoMD@gmail.com
}

How to cite this paper: Hellman, J. and Yao. J. (2019) Novel Histological Evidence of Collagen and Elastin Regeneration in Fractional RF-Treated Acne Scars. Journal of Cosmetics, Dermatological Sciences and Applications, 9, 155-164.

https://doi.org/10.4236/jcdsa.2019.92013

Received: April 1, 2019

Accepted: June 2, 2019

Published: June 5, 2019

Copyright () 2019 by author(s) and Scientific Research Publishing Inc. This work is licensed under the Creative Commons Attribution International License (CC BY 4.0).

http://creativecommons.org/licenses/by/4.0/

\begin{abstract}
Introduction: Fractional radiofrequency (RF) technology has been shown to be a gold standard therapy for acne scars. We have previously published clinical and histological results from the treatment of 8 patients with active acne and acne related scarring using a fractional RF device. Evidence of safety and efficacy was demonstrated, including long term follow-up clinical results from 4 patients, up to two years post last fractional treatment. In the current article we present histology evidence of acne scar skin renewal 3.5 years post treatment. Methods: Skin biopsies from acne scars in a concealed facial area were taken before, after four treatments and at 3.5 years after the last fractional treatment. Biopsies were examined with standard hematoxylin and eosin (H\&E) stain, as well as Verhoeff's Van Gieson (VVG) and Shikata stains for elastic fibers. Results: Histological findings demonstrate regeneration of elastic fibers and reduction of the dermal fibrosis. Scar depth of Patient \#2 was reduced by $80 \%$ in the corresponding follow-up period. Conclusion: The long-term histological results further support the previously published findings that fractional RF is a safe and effective treatment for acne related scars.
\end{abstract}

\section{Keywords}

Fractional Radiofrequency, Acne Scars, Histological Evidence, Long Term Follow-Up, Elastic Fibers Regeneration

\section{Introduction}

Permanent scarring from acne is an unfortunate prevalent complication of acne vulgaris which may be associated with considerable psychological distress. Dermatologists are frequently presented with the challenge of evaluating and pro- 
viding treatment recommendations to patients with acne scars [1].

Many different treatments, including chemical peels, surgical excision, punch grafting, dermabrasion, and tissue augmentation with a variety of filler substances, have been used to ameliorate atrophic scars with varying degrees of success [2] [3]. Recontouring of atrophic facial scars with $\mathrm{CO}_{2}$ and Er:YAG lasers has become popular over the last decade [4] [5]; however, due to the extended post-laser recovery period and the potential risks, especially to candidates with dark skin type, patients have been less willing to undergo ablative laser skin resurfacing [6].

Fractional resurfacing technologies using either $\mathrm{CO}_{2}$ fractional laser or fractional RF (FRF) energy delivered through multi-electrode pins, have demonstrated visible clinical benefits on atrophic acne scars and minor side effects comparing to non-fractional technologies [7].

In previously published articles we demonstrated our experience with an FRF device in the treatment of eight patients with active acne and acne-related scarring [8]. Long term clinical follow-up results of four out of eight patients who participated in the study were demonstrated, up to two years post last fractional treatment [9]. In the current publication we present long term histological evidence of acne scar skin renewal 3.5 years following completion of FRF treatment.

\section{Methods}

During the original study, all patients were informed about the risks, benefits and alternative treatments and all provided an informed consent based on the 1975 Declaration of Helsinki. Patients having acne scars, with or without active acne lesions, were treated with an array of $24 \mathrm{RF}$ conducting pins.

Each pin is $2500 \mu \mathrm{m}$ long (Fractora, InMode Ltd., Israel). As previously published, this study was originally carried out in the private clinic of Dr Judith Hellman in NY, NY. The treatment procedure was specified in the previously published articles [8] [9].

According to the typical treatment protocol, each patient underwent four facial treatments, usually four weeks apart. The first patient (PT1) - a male, 21 years old, with Fitzpatrick skin type II was treated with regular Fractora tip and the second patient (PT2) - a female, 19 years old, with Fitzpatrick skin type IV was treated with Fractora tip having pins with insulating coating along $2000 \mu \mathrm{m}$ that provided additional epidermal protection for dark skin.

Representative skin biopsies from two patients were taken before, after the fourth treatment and approximately 3.5 years after last treatment. The biopsies were taken from the same scarred areas of the face of each participant, to keep the results consistent, and the new biopsy was taken adjacent without overlapping to avoid the effect of the previous biopsy. Histological sections were fixed and examined with standard Hematoxylin and Eosin (H\&E) stain as well as with Verhoeff's elastic stain-(VEG) and Shikata stains for elastic fibers. In the interim period between the fourth treatment and the follow-up period, the patients 
did not undergo any treatment or use any topicals as the acne stopped breaking out and scars do not benefit from topicals.

\section{Results}

In a previously published article, histological sections of skin biopsies taken before treatment and after four fractional treatments from one patient were presented [8]. In the current publication, we compare baseline and results post four fractional treatments with histological sections from biopsies taken at 3.5 years after the last fractional treatment.

Figure 1 and Figure 2 demonstrate biopsies taken prior to treatment from two subjects. Figure 1 shows that the dermal fibrosis or acne scarring extends deep past the isthmus level (past the insertion of the pili erector muscles). The bracket highlights the depth of the dermal scar. In this area there is a loss of adnexal structures, notably the hair follicle. There are retained sebaceous glands beneath the fibrosis but the follicle is lost. The initial depth of the scarring process is $1.5 \mathrm{~mm}$ for PT1. It should be noted that in case of PT2, the pretreatment biopsy did not reflect the deepest part of the scar, only a depth of $0.50 \mathrm{~mm}$ was shown (Figure 2(b)). This may be due to sampling variants that occurred in the sectioning process of the histological slices.

Figure 2 shows biopsies performed prior to treatment stained with Shikata and VEG stains which are specific for elastic fibers. The Shikata preparation stains the elastic fibers black for PT1 and the VEG stain, a comparable elastic fibers silver stain, highlights elastic fibers in PT2 sample. Both histologies demonstrate dermal scar which replaces the normal elastic fibers and both stains clearly reveal areas with a loss of elastic fibers, replaced by fibrous tissue. In addition, the Shikata preparation of the biopsy demonstrates adjacent adnexal structures in the normal area with a drop out of adnexal structures in the scar tissue.

Figures 3-5 present PT1 outcome after four fractional treatments. Figure 3 demonstrates the reduction of scar depth to almost half for PT1 after four treatments. The maximal depth of scarring was measured as $0.8 \mathrm{~mm}$ vs. $1.5 \mathrm{~mm}$ before treatment.

In Figure 3 and Figure 4 regenerative near normalized wispy collagen fibers are present in the reticular dermis; the scar is reduced to the superficial dermis. In addition, elastic fibers that were devoid of the original scar tissue repopulated the treated area (Figure 5).

Figure 6 presents VEG stain of PT2 histology results after 4 treatments. In PT2, there appears to be sebaceous glands above the dermal scarring process as a result of adjacent reactive sebaceous hyperplasia. Below the sebaceous glands, there is clear dermal fibrosis with loss of elastic fibers which indicates definitive dermal fibrosis extending to depth measurement of $1.13 \mathrm{~mm}$. Of note, in the superficial dermis there are regenerative dystrophic elastics fibers, indicating elastic fiber regeneration and repopulation.

Figures 7-9 demonstrate the histologies taken 3.5 years post fractional treatments, revealing the maximum effect of fractional treatments and reduction of 
scar depth. Results show regeneration of elastic fibers that replace the dermal scar and reduce the dermal fibrosis.

Figure 7 shows the area of dermal scar stained with Shikata stain for PT1 and VEG stain for PT2. There is complete loss of elastin in the area marked by the circle. However, there are regenerative elastic fibers in the superficial papillary dermis above the dermal scar. Dermal scar depth was maximally reduced to 0.3 and $0.25 \mathrm{~mm}$ for PT1 and PT2, respectively. The dermal scar is replaced by near normal elastic fibers and reduction of the dermal fibrosis for both PT1 and PT2.

Figure 8 and Figure 9 represent the healing progress and the reduction in size of the acne scar following fractional treatments. Figure 8 demonstrates the healing progress of PT1 acne scar from a depth of $1.5 \mathrm{~mm}$ at baseline reduced to $0.8 \mathrm{~mm}$ after 4 treatments, and reduced up to $0.32 \mathrm{~mm}$ at 3.5 years follow-up. Figure 9 compares two biopsies of PT2.

Figure 10 and Figure 11 demonstrate the clinical improvement of both patients by comparing pretreatment photos and photos taken at 3.5 years post 4 Fractora treatments.

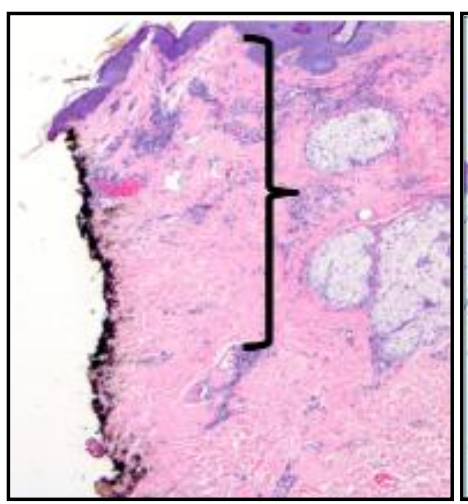

(a)

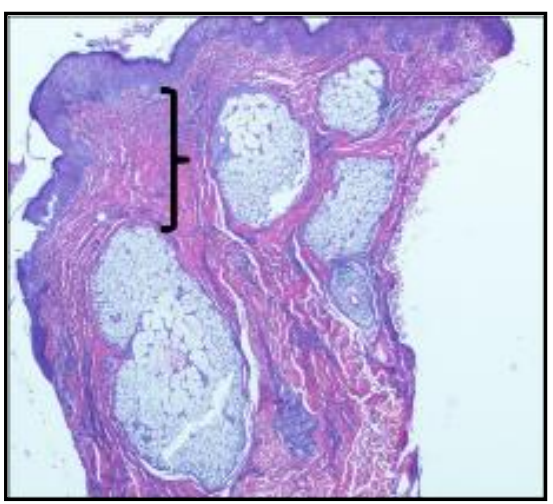

(b)

Figure 1. Histological section of skin biopsies from two representative patients before fractional treatments. PT1 histology (a) and PT2 (b) demonstrate acne scar tissue deep in the dermis, devoid of hair follicles and sebaceous glands. H\&E staining, $\times 4$.

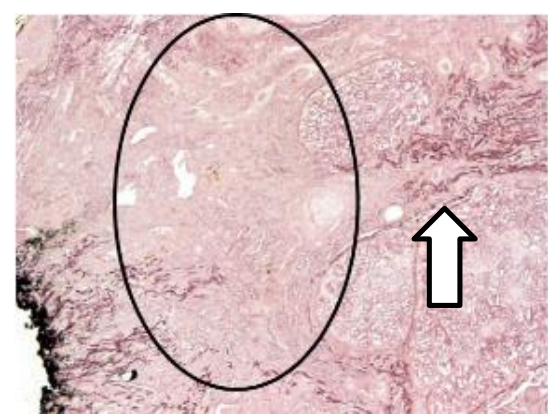

(a)

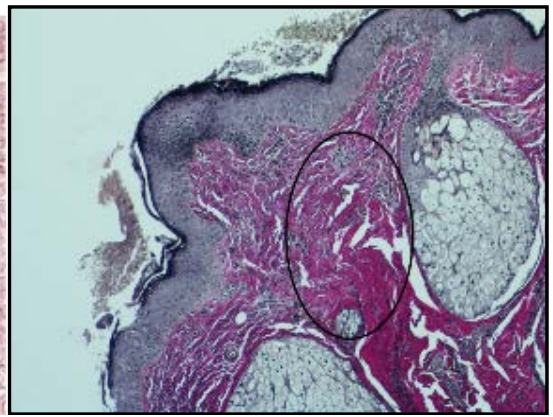

(b)

Figure 2. PT1 histology (a) demonstrates acne scar tissue mostly devoid of elastic fibers (circle), as opposed to normal elastic fibers pointed by arrow. Shikata staining, $\times 4$. PT2 histology (b) demonstrates the scar area with loss of elastic fibers, replaced by fibrous tissue(circle). VEG staining, $\times 4$. 


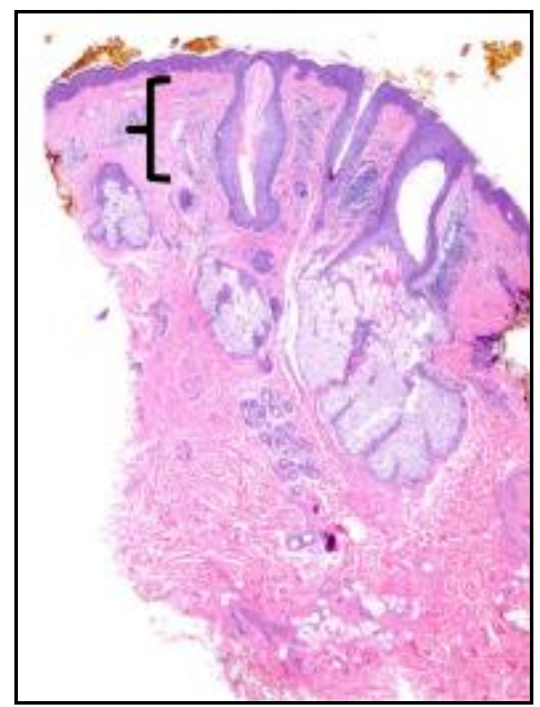

Figure 3. Scar regresses from $1.5 \mathrm{~mm}$ to $0.8 \mathrm{~mm}$ and new collagen reducing the original depth of the scar up. H\&E staining, $\times 4$.

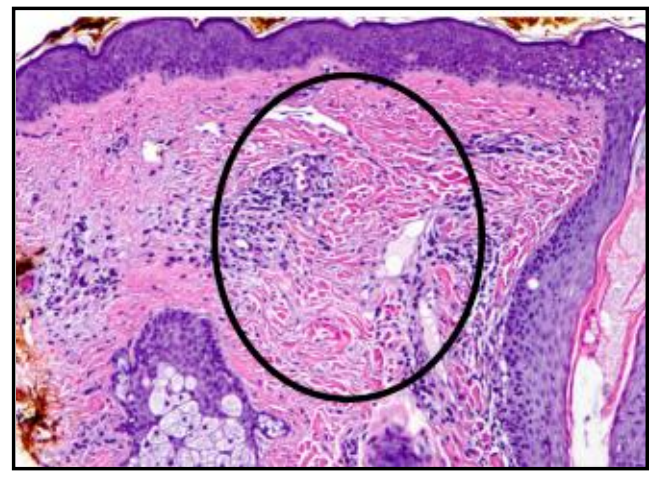

Figure 4. Higher magnification of area of dermal fibrosis H\&E staining, $\times 10$. There is disorganized collagen deposition with new small caliber vessel formation. This area of fibrosis is devoid of adnexal structures and blunted rete ridges, features of a dermal scar. The dermal scar is now reduced to the superficial dermis.

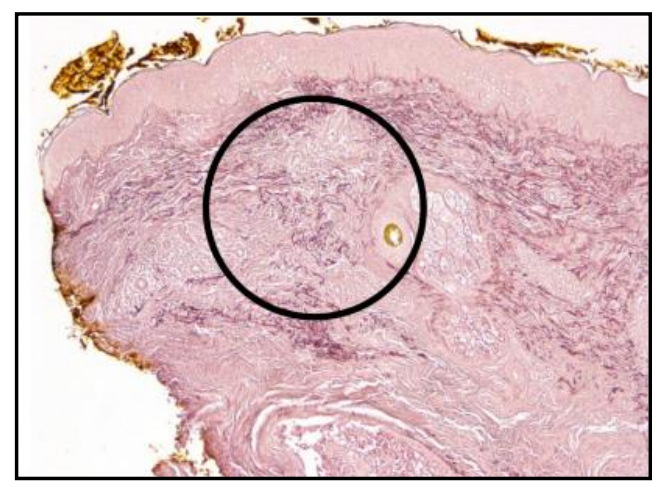

Figure 5. Area of scarring is denoted by loss of elastic fibers (dark staining by the Shikata stain) and also by loss of adnexal structures. Note the superficial papillary dermis and within the scarred area, there is a regeneration of few elastic fibers highlighted in the black circle. This illustrates an active scar reduction and scar correction, with the replacement of the dermal fibrosis with elastic fibers. 


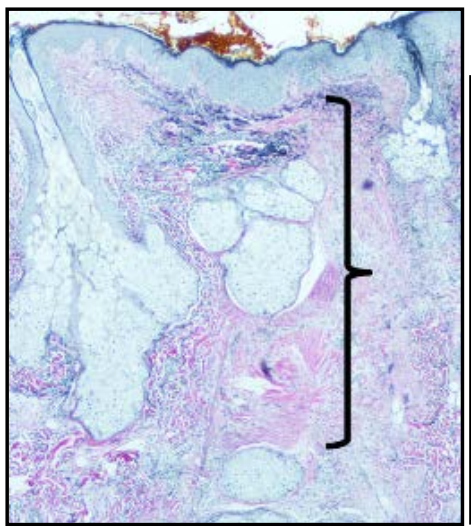

(a)

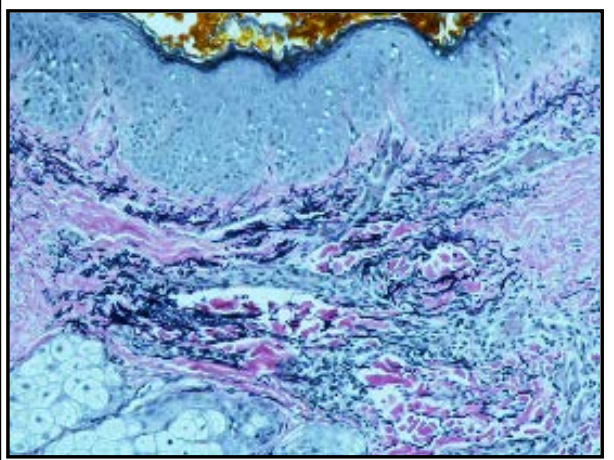

(b)

Figure 6. VEG stain showing dermal fibrosis below the sebaceous glands with loss of elastic fibers. In the superficial dermis regenerative dystrophic elastics fibers indicate elastic fiber regeneration and repopulation $(\mathrm{a} \times 4, \mathrm{~b} \times 10)$.

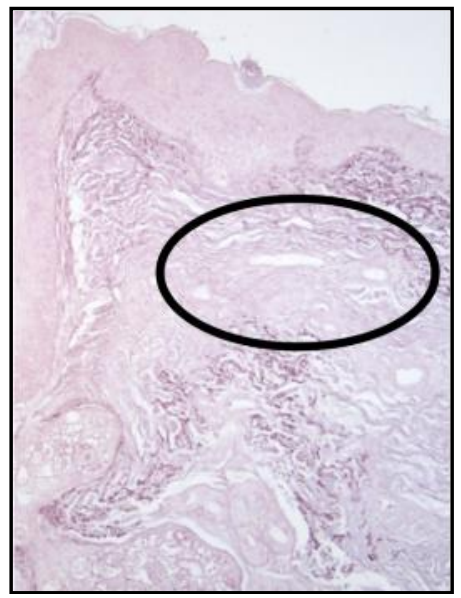

(a)

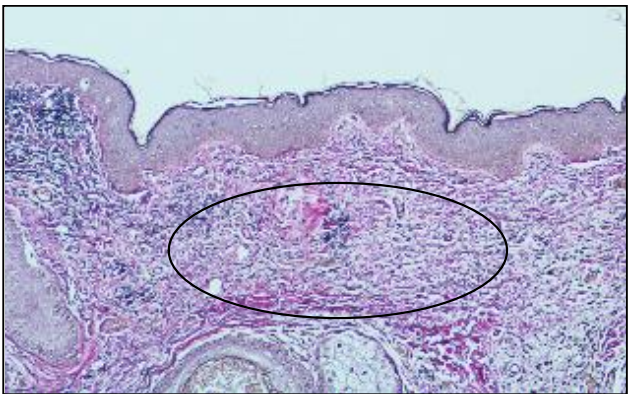

(b)

Figure 7. Area of dermal scar stained with the Shikata stain (a) and VEG stain (b). There is loss of elastin in the circled area but regenerative elastic fibers in the superficial papillary dermis above the dermal scar and near the circled area are clearly observed $(\times 10)$.

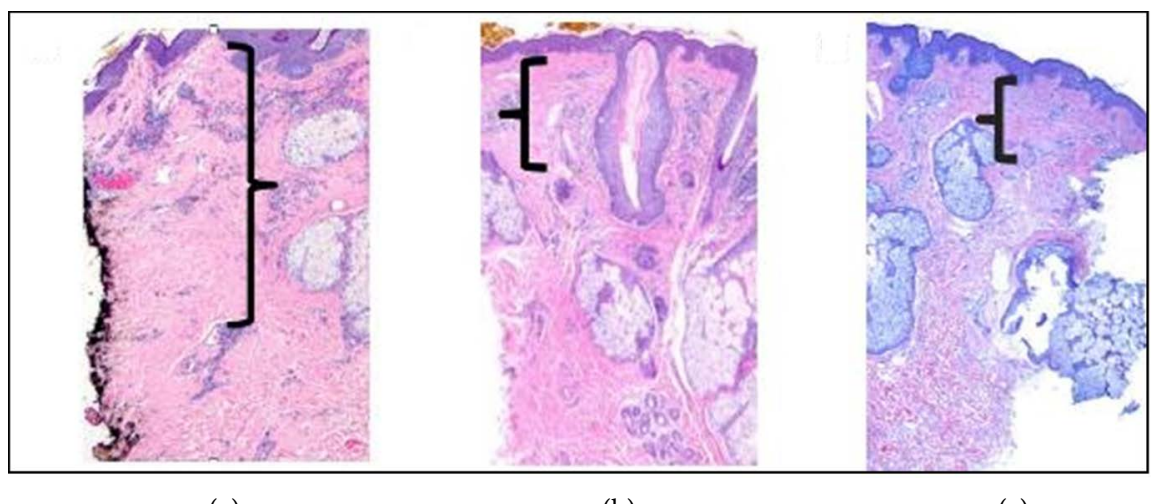

(a)

(b)

(c)

Figure 8. PT1 side by side comparison of the reduction of the acne scar. (a) Initial biopsy, prior to treatment, (b) after 4 treatments and (c) 3.5 years post fractional treatments. 


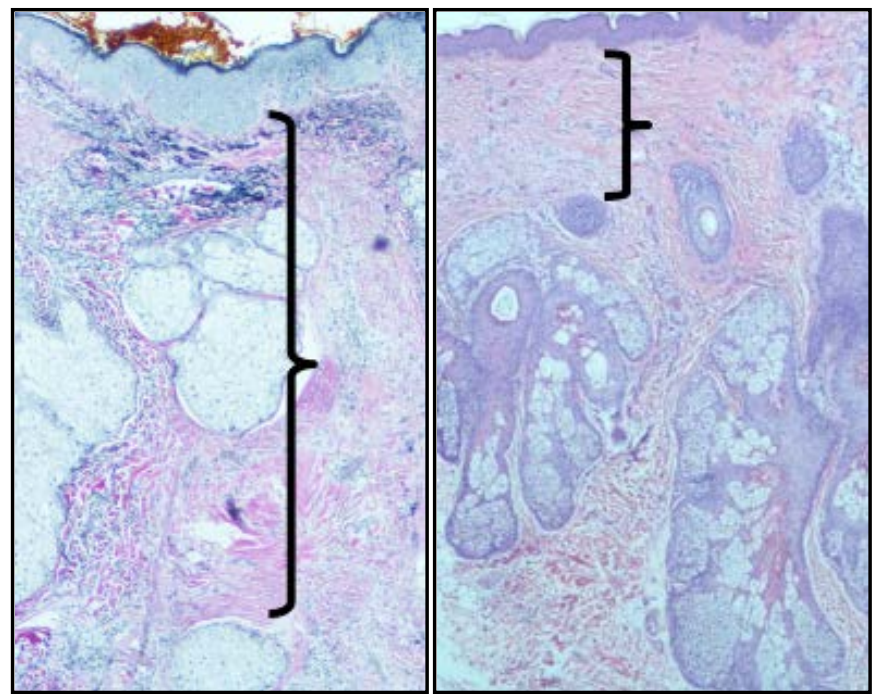

(a)

(b)

Figure 9. PT2 side by side comparison of the reduction of the acne scar: (a) after 4 treatments, (b) 3.5 years post fractional treatments.
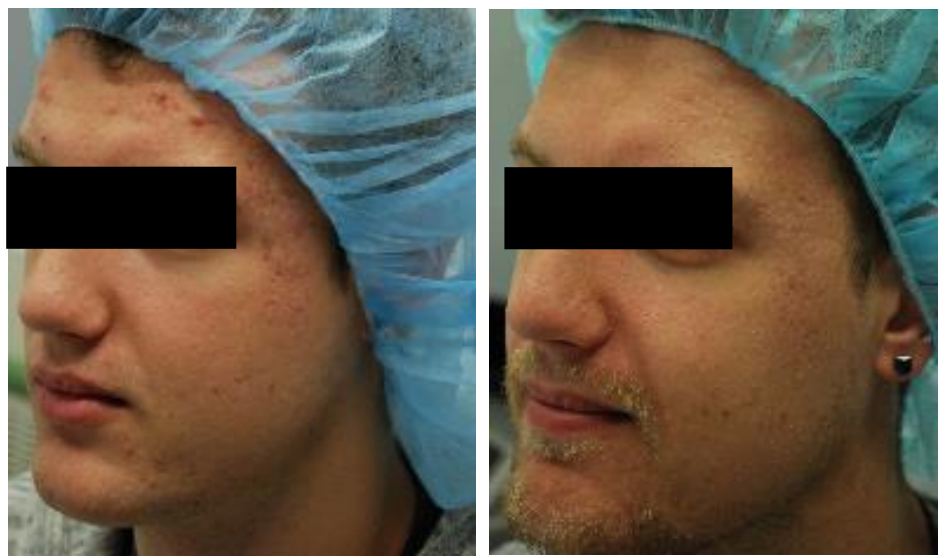

Figure 10. PT1 visual improvement of the acne scars appearance. (a) Baseline-before treatments, (b) 3.5 years post fractional treatments.
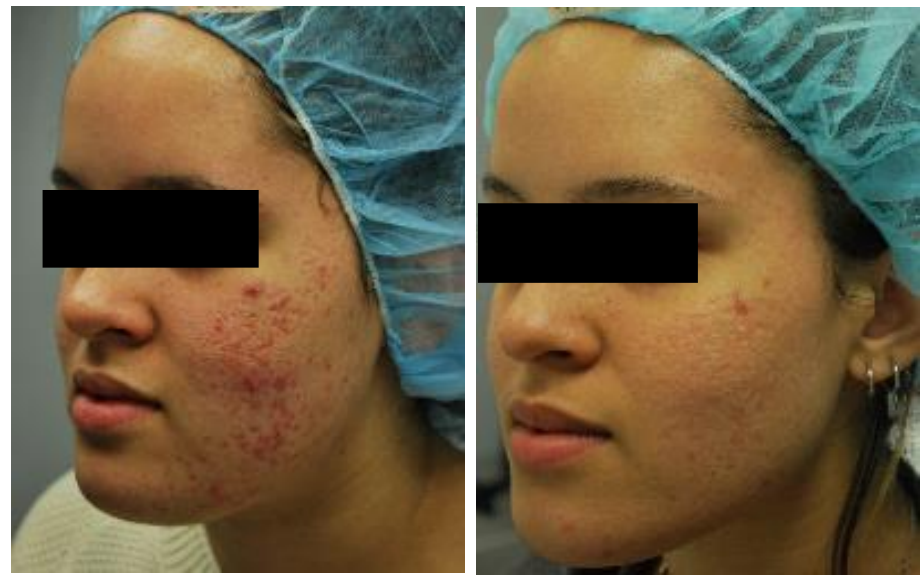

Figure 11. PT2 visual improvement of the acne scars appearance. (a) Baseline-before treatments, (b) 3.5 years post fractional treatments. 


\section{Discussion}

Our previous articles reported clinical and histological experience with the FRF device (Fractora, InMode, Ltd., Israel) for the treatment of active acne lesions and acne scarring [8] [9]. Treatment with FRF led to visible improvement in the appearance of the active acne and of acne scars. The depth of the scars was substantially reduced after four treatments and scars were regressed higher into the superficial dermis, apparently pushed up by new near normal collagen fibers. In addition, there was a repopulation of new elastic fibers in the superficial dermis previously occupied by scar tissue [8].

Long term clinical evidence was presented, demonstrating visual improvement of active acne lesions, acne scarring, pores and general skin texture after up to two years post last fractional treatment [9].

Long term follow-up histological results are not frequently available due to patient's compliance. In a study comparing a $1450 \mathrm{~nm}$ diode laser and a $1320 \mathrm{~nm}$ $\mathrm{Nd}$ :YAG laser in the treatment of atrophic facial scars, evaluations of cutaneous biopsies obtained before treatment, immediately after the first treatment, and at $1,3,6$, and 12 months after the third treatment were performed [10]. Histological evaluation demonstrated an increase in dermal collagen on both the $1320 \mathrm{~nm}$ Nd:YAG and $1450 \mathrm{~nm}$ diode laser treating split-face halves 6 months after the final laser procedure. Additional neocollagenesis was not observed at the 12-month follow-up visit [10]. In an earlier study, prolonged clinical and histological effects from $\mathrm{CO}_{2}$ laser resurfacing of atrophic acne scars were investigated [4]. Blinded histological analyses were made on skin biopsies immediately prior to and after laser resurfacing, and at 1, 6, 12, and 18 months postoperatively in six patients. Histopathology findings paralleled the clinical improvement seen. Specifically, the prolonged and enhanced clinical improvement correlated directly with the degree of new collagen deposition and dermal remodeling observed histologically up to and beyond 12 months. Continued neocollagenesis and subsequent dermal remodeling were observed on histological examination of biopsied tissue up to 18 months after surgery. In particular, 6-month postoperative specimens showed more densely packed collagen bundles. In addition, horizontal realignment of collagen fibers in the papillary dermis with increased collagen/elastin content, normalization in polarity of keratinocytes, and a return of the rete ridges were demonstrated. Investigators concluded that the histopathology results mirrored the three phases of wound repair: the inflammatory phase, the proliferative phase, and the remodeling phase [4] [11].

Treatment of active acne and of acne scars with FRF is safer compared to lasers since there is a shorter recovery time. Several studies employing variations of FRF devices have demonstrated the safety and efficacy of FRF technology in the treatment of acne scars [7] [8] [9] [12] [13] [14] [15]. In addition, the FRF microneedles penetrate the skin to target the RF energy deeper to the dermal layer on top of the external resurfacing effect [16]. 
The current publication demonstrates histological results 3.5 years post four fractional treatments of acne scars. Histological results of the reduction of acne scar depth support the ongoing visual long-term clinical results demonstrated previously [9]. Regeneration of collagen fibers and of elastic fibers was clearly observed in the current biopsies. In the case of PT1, an $80 \%$ reduction in scar depth was noted when comparing the depth measurement of $1.5 \mathrm{~mm}$ in pretreatment histologies with the follow-up measurement of $0.3 \mathrm{~mm}$ after 3.5 years. A similar depth reduction was noted in PT2 case, when comparing post four fractional treatments, the depth measurement of $1.13 \mathrm{~mm}$ to the follow-up measurement of $0.25 \mathrm{~mm}$ after 3.5 years. Comparison to baseline was not possible since accurate pretreatment histology was not available. This was due to sectioning angle variations, leading to a sampling error in which the biopsy was not representative of the deepest dermal scar. It is postulated though, that the pretreatment scar depth was even deeper than the post four treatments measurement. Even without such an assumption, however, there was a visible clinical improvement in the appearance of the acne scars, reflecting the $80 \%$ reduction in scar depth in the case of PT2.

The finding of the substantial improvement in scar depth, and the fact that new elastic fibers are regenerated, may be helpful in treating various indications caused by irreversible elastin damage such as scarring alopecia, solar elastosis and smokers' wrinkles. In the latter example, free radicals destroy the elastic fibers and activate elastase, thus regeneration of elastic fibers may have a profound efficacy for the treatment of such indications.

It should be noted that in order to scientifically support these initial histological findings, more cases should be followed similarly for a long period after treatment. Results from more subjects will substantiate the safety and efficacy of the FRF modality for treatment of acne scars.

\section{Conclusion}

Skin biopsies clearly demonstrate the efficacy of the Fractora treatment. Substantial reduction of acne scar depth and repopulation of the scar tissue by collagen and elastic fibers are visible 3.5 years after fractional treatments.

\section{Conflicts of Interest}

The authors declare no conflicts of interest regarding the publication of this paper.

\section{References}

[1] Fife, D. (2011) Practical Evaluation and Management of Atrophic Acne Scars Tips for the General Dermatologist. The Journal of Clinical and Aesthetic Dermatology, 4, 50-57.

[2] Alster, T.S. and West, T.B. (1997) Treatment of Scars: A Review. Annals of Plastic Surgery, 39, 418-432. https://doi.org/10.1097/00000637-199710000-00014 
[3] Alster, T.S. and Tanzi, E.L. (2003) Hypertrophic Scars and Keloids: Etiology and Management. American Journal of Clinical Dermatology, 4, 235-243. https://doi.org/10.2165/00128071-200304040-00003

[4] Walia, S. and Alster, T.S. (1999) Prolonged Clinical and Histologic Effects from $\mathrm{CO}_{2}$ Laser Resurfacing of Atrophic Acne Scars. Dermatologic Surgery, 25, 12. https://doi.org/10.1046/j.1524-4725.1999.99115.x

[5] Tanzi, E.L. and Alster, T.S. (2002) Treatment of Atrophic Acne Scars with a Dual Mode Er:YAG Laser. Dermatologic Surgery, 28, 551-555. https://doi.org/10.1046/j.1524-4725.2002.01319.x

[6] Nanni, C.A. and Alster, T.S. (1998) Complications of $\mathrm{CO}_{2}$ Laser Resurfacing: An Evaluation of 500 Patients. Dermatologic Surgery, 24, 315-320. https://doi.org/10.1111/j.1524-4725.1998.tb04161.x

[7] Zhang, Z., Fei, Y., Chen, X., et al. (2014) Comparison of a Fractional Microplasma Radio Frequency Technology and Carbon Dioxide Fractional Laser for the Treatment of Atrophic Acne Scars: A Randomized Split-Face Clinical Study. Journal of Cutaneous and Aesthetic Surgery, 7, 93-97.

[8] Hellman, J. (2015) Retrospective Study of the Use of a Fractional Radio Frequency Ablative Device in the Treatment of Acne Vulgaris and Related Acne Scars. Journal of Cosmetics, Dermatological Sciences and Application, 5, 311-316. https://doi.org/10.4236/jcdsa.2015.54038

[9] Hellman, J. (2016) Long Term Follow-Up Results of a Fractional Radio Frequency Ablative Treatment of Acne Vulgaris and Related Acne Scars. Journal of Cosmetics, Dermatological Sciences and Application, 6, 100-104. https://doi.org/10.4236/jcdsa.2016.63013

[10] Tanzi, E.L. and Alster, T.S. (2004) Comparison of a 1450-nm Diode Laser and a 1320-nm Nd:YAG Laser in the Treatment of Atrophic Facial Scars: A Prospective Clinical and Histologic Study. Dermatologic Surgery, 30, 152-157. https://doi.org/10.1111/j.1524-4725.2004.30078.x

[11] Kirsner, R.S. and Eaglestein, W.H. (1993) The Wound Healing Process. Dermatologic Clinics, 11, 629-640. https://doi.org/10.1016/S0733-8635(18)30216-X

[12] Forbat, E. and Al-Niaimi, F. (2016) Fractional Radiofrequency Treatment in Acne Scars: Systematic Review of Current Evidence. Journal of Cosmetic and Laser Therapy, 18, 442-447. https://doi.org/10.1080/14764172.2016.1225964

[13] Min, S., Park, S.Y., et al. (2016) Fractional Micro-Needling Radiofrequency Treatment for Acne-Related Post-Inflammatory Erythema. Acta Dermato-Venereologica, 96, 87-91. https://doi.org/10.2340/00015555-2164

[14] Verner, I. (2016) Clinical Evaluation of the Efficacy and Safety of Fractional Bipolar Radiofrequency for the Treatment of Moderate to Severe Acne Scars. Dermatologic Therapy, 29, 24-27. https://doi.org/10.1111/dth.12275

[15] Lan, T., Xiao, Y., Tang, L., Hamblin, M.R. and Yin, R. (2018) Treatment of Atrophic Acne Scarring with Fractional Micro-Plasma Radio-Frequency in Chinese Patients: A Prospective Study. Lasers in Surgery and Medicine, 50, 844-850. https://doi.org/10.1002/lsm.22825

[16] Mulholland, R.S., Ahn, D.H., Kreindel, M. and Paul, M. (2012) Fractional Ablative Radio-Frequency Resurfacing in Asian and Caucasian Skin: A Novel Method for Deep Radiofrequency Fractional Skin Rejuvenation. Journal of Cosmetics, Dermatological Sciences and Applications, 2, 144-150.

https://doi.org/10.4236/jcdsa.2012.23029 